Available online at GSC Online Press Directory

GSC Biological and Pharmaceutical Sciences

e-ISSN: 2581-3250, CODEN (USA): GBPSC2

Journal homepage: https://www.gsconlinepress.com/journals/gscbps

(RESEARCH ARTICLE)

\title{
In silico studies of pyrano [2, 3-c] pyrazoles derivatives as cyclooxygenase-2 inhibitiors
}

\author{
Bais G Abhinav*, Chaple R Dinesh, Asnani J Alpana, Kumar Pratyush and Mohurle Shrikant \\ Priyadarshini J. L. College of Pharmacy, Electronic zone, Electronic building, Hingna Road, Nagpur-440016, Maharashtra, \\ India.
}

Publication history: Received on 10 April 2020; revised on 15 April 2020; accepted on 16 April 2020

Article DOI: https://doi.org/10.30574/gscbps.2020.11.1.0098

\begin{abstract}
Non-steroidal anti-inflammatory drugs (NSAID's) have been used widely from several decades for treatment of analgesia and inflammation. The most widely reported side effect of NSAID's is inflammation of gastric regions, ulceration and kidney problems. These side effects are due to non-selective inhibition of cyclooxygenase-2 over cyclooxygenase-1.Therefore we planned to design a potent cyclooxygenase-2 inhibitor using insilico techniques which may be used as anti-inflammatory and analgesics. In this current study we have chosen Pyrano[2,3-c] pyrazoles as the parent moiety along with several derivatives. These will acts as ligand molecules for computational protocols. The crystalline structure of cyclooxygenase- 2 was downloaded from protein database and the pdb code was $1 \mathrm{cx} 2$. This will act as target for computational studies. Pyrx software was used for virtual screening of library of derivatives. The molecular docking of potent derivatives were carried using autodock software X:Y:Z (50:26:40). Other insilico properties were calculated using Molinspiration online property calculator, Protox II for structural property calculation and acute oral toxicity determination respectively. Derivatization in the molecule is must for increasing biological potential of parent moiety. The study revealed best molecule that was having potent analgesic and anti-inflammatory activity. Results revealed though the ligand molecule was safe and effective for cyclooxygenase-2 inhibition. The LD50 calculated was found to be $500 \mathrm{mg} / \mathrm{kg}$. Other In silico property were also calculate.
\end{abstract}

Keywords: NSAID’s; Cyclooxygenase-2 inhibitor; Pyrx software; Autodock software

\section{Introduction}

Non-steroidal anti-inflammatory drugs (NSAID's) have been used widely from several decades for treatment of analgesia and inflammation. The most widely reported side effect of NSAID's is inflammation of gastric regions, ulceration and kidney problems. These side effects are due to non-selective inhibition of cyclooxygenase- 2 over cyclooxygenase-1.

The heterocyclic compounds are widely spread in nature and play an important role in life. Due to the characteristic properties, the heterocyclic compounds hold a large area in medicinal chemistry [1]. The chemistry of heterocyclic chemistry has been explored widely in the past two - three decades [2]. The synthesis and the application of heterocyclic compounds of medium size rings became popular [3,4]. During the recent years, there has been intense research on fused heterocyclic compounds with pharmacological importance. Among the heterocyclic of pyranopyrazole class has drawn the attention. Pyranopyrazoles refer to a fused five member pyrazole ring to a six member pyran ring. The pyranopyrazole nucleus is a versatile source of biologically important molecules. There are four possible isomers of pyranopyrazole-pyrano[2,3-c]pyrazole, pyrano[4,3-c]pyrazole, pyrano[3,2-c]pyrazole and pyrano[3,4-c]-pyrazole.

\footnotetext{
* Corresponding author: Bais G Abhinav
} 


\subsection{Objective}

In the current research we to screen the binding efficiency of pyrano[2,3-c] pyrazoles derivatives with cyclooxygenase2 receptor and predict structural and biological properties of ligand.

\section{Material and methods}

\subsection{Softwares and programs}

Chemsketch a chemical molecule drawing tool was used to draw the ligand compounds [5]. Avogadro software was used to convert the .mol file to .pdb format [6]. Autodock 4.0 [7] a preliminary docking program was used for the semi-flexible protein ligand docking studies. Molinspiration online property calculator was used to study the chemical properties of the compound [8]. The crystalline structure of cyclooxygenase- 2 was downloaded from protein database and the pdb code was [PDB: 1cx2]. This will act as target for computational studies. Pyrx software was used for virtual screening of library of derivatives. [9] Discovery studio 3.5 was used for molecular interaction and visualization.[10].

\subsection{Preparation of ligand}

Ligand structure was drawn using Chemsketch software and the structure was cleaned using the clean structure tool. The structure was saved in the working folder as .mol file. The .mol file was then accessed in Avogadro software and structure was optimized using optimization tool. The optimized structure was saved in the working directory as .pdb file.

\subsection{Preparation of receptor}

The crystal structure of anti-inflammatory was downloaded in .pdb format from the online database and was rectified using Autodock v4.0 software. The energy was minimized by spreading the charges all over the receptor. The water molecules associated with receptor was deleted and polar hydrogen molecules were added.

\subsection{Receptor-Ligand Docking}

Autodock v4.0 was used to identify binding poses with associated binding energies. As per the inverse relation of energy and stability, the conformation with greater binding energy is less stable. The Default parameters of the software program have been applied similar to the protocol followed elsewhere [26-29]. Briefly, Lamarckian Genetic Algorithm (LGA) [30] with default atomic salvation parameters $126 \AA$ ( $\mathrm{x}, \mathrm{y}$, and z) grid box in ratio of (60:60:60) for scoring energy was set at co-ordinates as $\mathrm{X}=50, \mathrm{Y}=26$ and $\mathrm{Z}=40$ with 0.375 angstroms grid points spacing. Care was given during the grid box preparation to ensure that the active site of receptor was surrounded by the 3D grid box centered at its active ligand binding site location.

\subsection{Online chemical property calculator}

Molinspiration online property calculator [7] was used for calculating the properties of the ligand. The structure of the ligand was drawn using inbuilt tool and several properties were calculated. The properties were classified broadly into two types as structural property and bioactivity. Acute oral toxicity was predicted using Protox II web server. [11,12].

\section{Results and discussion}

The structure of ligand and receptor was critically studied and drawn through insilico tools fig.1. The grid box of X:Y:Z (50:26:40) was made using Autodock v4.0 and is represented as in fig.2. 
<smiles>Cc1n[nH]c2c1C(C#N)C(C#N)=C(N)O2</smiles>

Figure 1 Structure of ligand
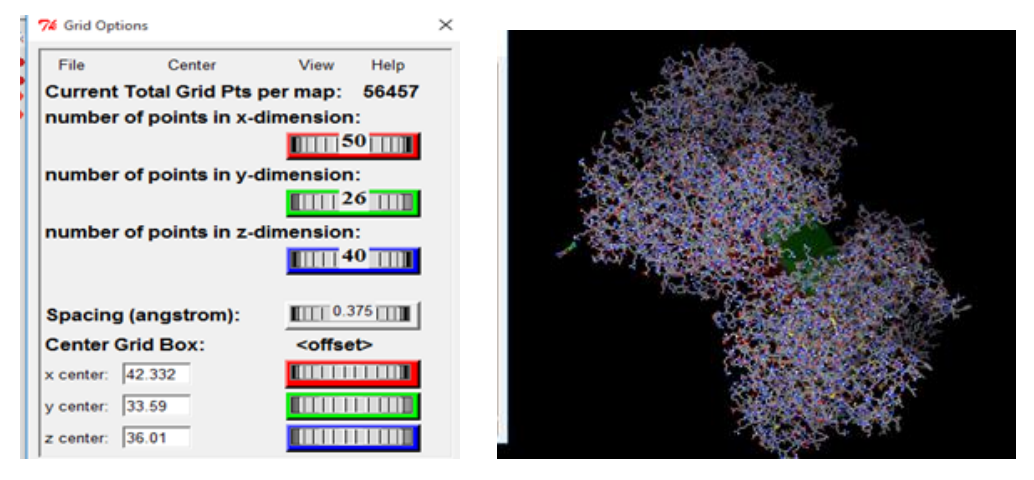

Figure 2 b: Structure of receptor; c: Grid box (6-amino-3-methyl-1,4- dihydropyrano[2,3-c]pyrazole-5-carbonitrile)

The result of docking Fig 3 shows that proposed ligand was adequately bound at the centre of the grid. On study of molecular interactions the amino acids with which ligand was showing interactions were TRP114; TYR149; ILE152; PR0155; LEU162; ASN397; PHE320; ASP401; LEU400; which is represented in Fig 4.

The mean binding energy of the best conformation was found to be -7.5 and the root mean square deviation (RMSD) value was found to be 11.75 . The free energy was found to be $-1345.90 \mathrm{Kcal} / \mathrm{mol} 11$.

The results of molinspiration online property calculator were represented as structural property and predicted bioactivity in table 1.

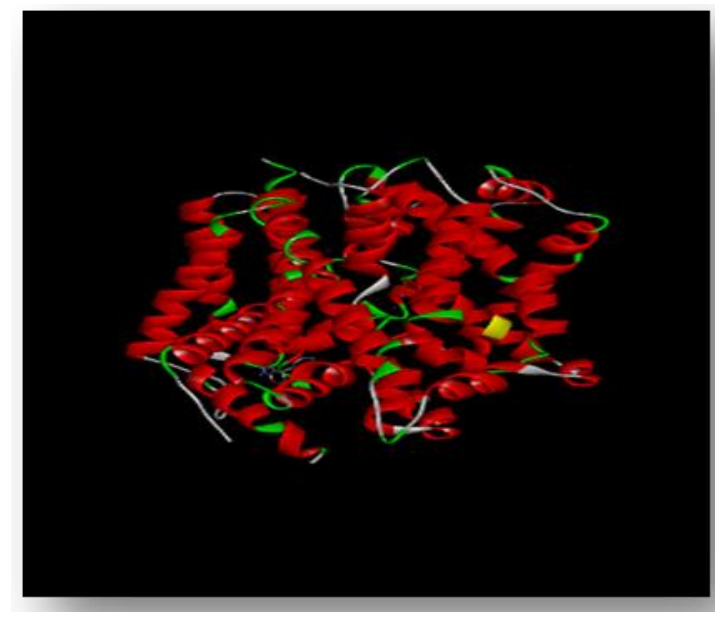

Figure 3 Docking of ligand with receptor

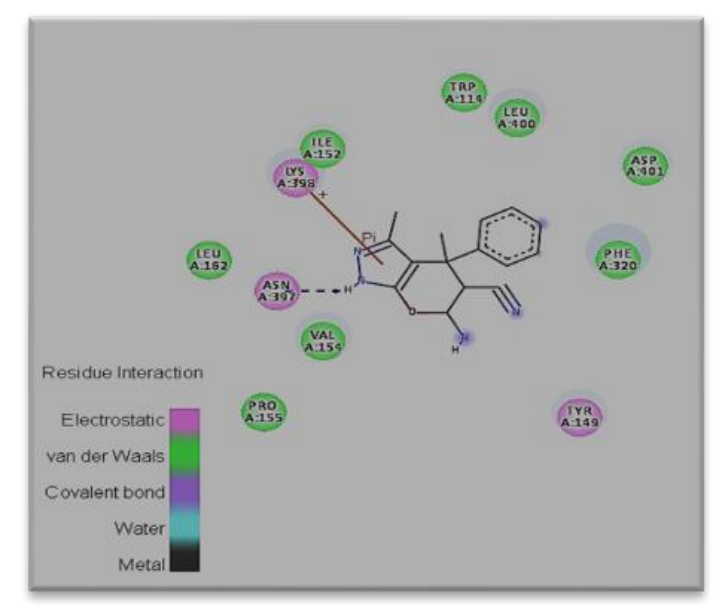

Figure 4 Molecular interactions of ligand with target 
Table 1 Results of Binding Affinity of Molecule

\begin{tabular}{llll}
\hline Mode & Affinity & Dist. From rmsd L. b. & Best for rmsd u.b. \\
\hline 1 & (Kcal/mol) & & \\
\hline 2 & -7.5 & 0.00 & 0.00 \\
3 & -7.3 & 8.829 & 11.715 \\
4 & -7.2 & 10.768 & 12.553 \\
5 & -7.1 & 11.575 & 13.957 \\
6 & -7.1 & 12.590 & 15.132 \\
7 & -7.0 & 11.291 & 13.878 \\
8 & -7.0 & 8.334 & 10.978 \\
9 & -6.9 & 9.467 & 11.839 \\
\hline
\end{tabular}

Table 2 Results of Molinspiration online property calculator

\begin{tabular}{ccccc}
\hline Sr. No & \multicolumn{2}{c}{ Structural Property } & \multicolumn{2}{c}{ Predicated Bioactivity } \\
& Property & Value & Site & Binding efficiency \\
\hline 1 & miLog p & 0.23 & GPCR Ligand & -1.29 \\
2 & TPSA & 87.73 & Ion Channel modulator & -0.92 \\
3 & Natoms & 13 & Kinase Inhibitor & -1.32 \\
4 & MW & 178.19 & Nuclear receptor ligand & -2.01 \\
5 & nON & 5 & Protease Inhibitor & -1.46 \\
6 & nOHNH & 3 & Enzyme Inhibitor & -1.01 \\
7 & Volume & 158.18 & & \\
\hline
\end{tabular}

\section{Conclusion}

The study showed Pyrano[2,3-c]pyrazole(6-amino-3-methyl-1,4-dihydropyrano[2,3-c]pyrazole-5-carbonitrile) molecule having potent analgesic and anti-inflammatory activity. The Results revealed though the ligand molecule was safe and effective for cyclooxygenase- 2 inhibition. The LD 50 Calculated was found to be $500 \mathrm{mg} / \mathrm{kg}$ and other physicochemical properties were in range.

In Silico Study gave Physicochemical properties and predicted bioactivity of molecule which will elaborated for further studies.. 


\section{Compliance with ethical standards}

\section{Acknowledgments}

The authors gratefully acknowledge the support provided by Principal and HOD and Teaching Staff of Priyadarshini J.

L. College of Pharmacy, Nagpur.

\section{Disclosure of conflict of interest}

The authors declare that there are no conflicts of interest.

\section{References}

[1] Pozharskii Af, Sodatemkov At and Kartritzky Ar. (1997). Heterocycles in Life and Society: An Introduction to Heterocyclic Chemistry, Biochemistry and Applications, Second Edition, 6(4), 301.

[2] Jug K, Oricine Dc and Katritzky Ar. (2001). Quantitative Measures of Aromaticity for Mono-, Bi-, and Tricyclic Penta and Hexaatomic Hetero aromatic Ring Systems and Their Interrelationships, Chemistry Review, 101, 14211450.

[3] Elnagdi Mh, Elmoghayar Mrh and Sadek Ku. (1987). Chemistry of Pyrazolopyrimidines, Advance Heterocyclic Chemistry, 41, 314.

[4] Elnagdi Mh, Elmoghayar Mrh and Sadek Ku. (1990). Chemistry of Pyrazolopyrimidines, Advance Heterocyclic Chemistry, 48, 223.

[5] Chemsketch. (2014).

[6] Avogadro. (2010).

[7] Autodock v4.0. (2014).

[8] Molinspiration online property calculator. (2018).

[9] Pyrex. (2010).

[10] Discovery studio 3.5. (2011).

[11] Banerjee P, Eckert OA, Schrey AK and Preissner R. (2018). ProTox-II: a webserver for the prediction of toxicity of chemicals. Nucleic Acids Res (Web server issue 2018); NAR.

[12] Kumar P and Asnani A. (2019). Docking of 3,5-diphenyl- pyrazoline with monoamine oxidase A receptor and InSilico structural property calculation, Journal of Drug Delivery \& Therapeutics, 9(3-s), 43-45.

\section{How to cite this article}

Bais GA, Chaple RD, Asnani JA, Kumar P and Mohurle S. (2020). In silico studies of pyrano[2,3-c] pyrazoles derivatives as cyclooxygenase-2 inhibitiors. GSC Biological and Pharmaceutical Sciences, 11(1), 166-170. 